\title{
Algebraic General Solutions of Algebraic Ordinary Differential Equations
}

\author{
J.M. Aroca and J. Cano \\ Department of Algebra, Geometry and Topology \\ Fac. Ciencias. Univ. de Valladolid \\ Valladolid 47011, Spain \\ (aroca,jcano)@agt.uva.es
}

\author{
Ruyong Feng and Xiao-Shan Gao \\ Key Laboratory of Mathematics Mechanization \\ Institute of Systems Science, AMSS, \\ Academia Sinica, Beijing 100080, China \\ ryfeng@amss.ac.cn \\ xgao@mmrc.iss.ac.cn
}

\begin{abstract}
In this paper, we give a necessary and sufficient condition for an algebraic ODE to have an algebraic general solution. For an autonomous first order ODE, we give an optimized bound for the degree of its algebraic general solutions and a polynomial-time algorithm to compute an algebraic general solution if it exists.
\end{abstract}

\section{INTRODUCTION}

Finding the close form solution of an ODE can be traced back to the work of Liouville. For the algorithm consideration, the pioneer work is due to Risch. In [17], Risch gave an algorithm to find the elementary integration of $\int u d x$ where $u$ is a rational function or a monomial over $\mathbf{Q}(x)$. In Trager's Ph.D thesis [22], he gave a method to compute the integration of algebraic functions. In [1], Bronstein generalized Trager's results to elementary functions. For higher order linear homogeneous ODEs, Kovacic presented an effective method to find the Liouvillian solutions for second order ODEs [14]. In [19], Singer established a general framework for finding the Liouvillian solutions for general linear homogeneous ODEs. Many other interesting results on finding the Liouvillian solutions of linear ODEs were reported in $[2,6,23,24]$.

Most of these results are limited to the linear case or some special type nonlinear equations. Work on finding closed form solutions for nonlinear differential equations is not systematic as that for linear equations. With respect to the particular ODEs of the form $y^{\prime}=R(x, y)$ where $R(x, y)$ is a rational function, Darboux and Poincaré made important contributions [16]. More recently, Cerveau, Carnicer and Corral et al also made important progresses [4, 3, 7]. In particular, Carnicer gave the degree bound of algebraic solutions in the nondicritical case. In [20], Singer studied the Liouvillian first integrals of differential equations. In [12], Hubert gave a method to compute a basis of the general so- lutions of first order ODEs and applied it to study the local behavior of the solutions. In [9, 10], Feng and Gao gave a necessary and sufficient condition for an algebraic ODE to have a rational type general solution and a polynomial-time algorithm to compute a rational general solution if it exists.

In this paper, the idea proposed in [9] is generalized to compute the algebraic function solutions. In Section 2, we give a sufficient and necessary condition for an algebraic ODE to have an algebraic general solution, by constructing a class of differential equations whose solutions are all algebraic functions. In Section 3, by treating the variable and its derivative as independent variables, a first order autonomous ODE defines an algebraic plane curve. Using Riemann-Hurwitz formula, we give a degree bound of the algebraic function solutions of the equation. This degree bound is optimized in the sense that there is a class of first order autonomous ODEs, whose algebraic function solutions reach this bound. In Section 4, based on the above results and the theory of Hermite-Padé approximants, we give a polynomial-time algorithm to find an algebraic general solution for a first order autonomous ODE.

A first order autonomous ODE $F\left(y, \frac{d y}{d x}\right)=0$ can be reduced to the form $G\left(y, \frac{d x}{d y}\right)=0$, where $G$ is also a polynomial. Then to find the solution of $F=0$, we may first find $x=\phi(y)$ as a function in $y$ by computing the integration of an algebraic function, and then compute the inversion $y=\phi^{-1}(x)$. Hence, our algorithm is equivalent to a polynomial-time algorithm for finding an algebraic integration for algebraic functions. The previously known algorithms for elementary function integration have exponential worst-case complexity.

\section{ALGEBRAIC GENERAL SOLUTIONS OF ALGEBRAIC ODES}

\subsection{Definition of algebraic general solutions}

In the following, let $\mathbf{K}=\mathbf{Q}(x)$ be the differential field of rational functions in $x$ with differential operator $\frac{\mathrm{d}}{\mathrm{d} x}$ and $y$ an indeterminate over $\mathbf{K}$. Let $\overline{\mathbf{Q}}$ be the algebraic closure of the rational number field $\mathbf{Q}$. We denote by $y_{i}$ the $i$-th derivative of $y$. We use $\mathbf{K}\{y\}$ to denote the ring of differential polynomials over the differential field $\mathbf{K}$, which consists of the polynomials in the $y_{i}$ with coefficients in $\mathbf{K}$. All differential polynomials in this paper are in $\mathbf{K}\{y\}$. Let $\Sigma$ be a system of differential polynomials in $\mathbf{K}\{y\}$. A zero of $\Sigma$ is 
an element in a universal extension field of $\mathbf{K}$, which vanishes every differential polynomial in $\Sigma$ [18]. In this paper, we also assume that the universal extension field of $\mathbf{K}$ contains infinite number of arbitrary constants. We will use $\mathcal{C}$ to denote the constant field of the universal extension field of $\mathbf{K}$.

Let $P \in \mathbf{K}\{y\} / \mathbf{K}$. We denote by $\operatorname{ord}(P)$ the highest derivative of $y$ in $P$, called the order of $P$. Let $o=\operatorname{ord}(P)>0$. We may write $P$ as follows

$$
P=a_{d} y_{o}^{d}+a_{d-1} y_{o}^{d-1}+\ldots+a_{0}
$$

where $a_{i}$ are polynomials in $y, y_{1}, \ldots, y_{o-1}$ and $a_{d} \neq 0$. $a_{d}$ is called the initial of $P$ and $S=\frac{\partial P}{\partial y_{o}}$ is called the separant of $P$. The $k$-th derivative of $P$ is denoted by $P^{(k)}$. Let $S$ be the separant of $P, o=\operatorname{ord}(P)$ and an integer $k>0$. Then we have

$$
P^{(k)}=S y_{o+k}+R_{k}
$$

where $R_{k}$ is of lower order than $o+k$.

Let $P$ be a differential polynomial of order $o$. A differential polynomial $Q$ is said to be reduced with respect to $P$ if $\operatorname{ord}(Q)<o$ or $\operatorname{ord}(Q)=o$ and $\operatorname{deg}\left(Q, y_{o}\right)<\operatorname{deg}\left(P, y_{o}\right)$. For two differential polynomials $P$ and $Q$, let $R=\operatorname{prem}(P, Q)$ be the differential pseudo-remainder of $P$ with respect to $Q$. We have the following differential remainder formula for $R$ $[13,18]$

$$
J P=\sum_{i} B_{i} Q^{(i)}+R
$$

where $J$ is a product of certain powers of the initial and separant of $Q$ and $B_{i}, R$ are differential polynomials. Moreover, $R$ is reduced with respect to $Q$. For a differential polynomial $P$ with order $o$, we say that $P$ is irreducible if $P$ is irreducible when $P$ is treated as a polynomial in $\mathbf{K}\left[y, y_{1}, \ldots, y_{o}\right]$.

Let $P \in \mathbf{K}\{y\} / \mathbf{K}$ be an irreducible differential polynomial and

$$
\Sigma_{P}=\{A \in \mathbf{K}\{y\} \mid S A \equiv 0 \bmod \{P\}\} .
$$

where $\{P\}$ is the perfect differential ideal generated by $P[13$, 18]. Ritt proved that [18]

LEMma 2.1. $\Sigma_{P}$ is a prime differential ideal and a differential polynomial $Q$ belongs to $\Sigma_{P}$ iff $\operatorname{prem}(Q, P)=0$.

Let $\Sigma$ be a non-trivial prime ideal in $\mathbf{K}\{y\}$. A zero $\eta$ of $\Sigma$ is called a generic zero of $\Sigma$ if for any differential polynomial $P, P(\eta)=0$ implies that $P \in \Sigma$. It is well known that an ideal $\Sigma$ is prime iff it has a generic zero [18].

As a consequence of Lemma 2.1, we have

Lemma 2.2. Let $F \in \mathbf{K}\{y\} / \mathbf{K}$ be an irreducible differential polynomial with a generic solution $\eta$. Then for a differential polynomial $P$ we have $P(\eta)=0$ iff $\operatorname{prem}(P, F)=0$.

The following definition of general solution is due to Ritt.
Definition 2.3. Let $F \in \mathbf{K}\{y\} / \mathbf{K}$ be an irreducible differential polynomial. A general solution of $F=0$ is defined as a generic zero of $\Sigma_{F}$. An algebraic general solution of $F=0$ is defined as a general solution $\hat{y}$ which satisfies the following equation

$$
G(x, y)=\sum_{j=0}^{n} \sum_{i=0}^{m_{j}} a_{i, j} x^{i} y^{j}=0
$$

where $a_{i, j}$ are in $\mathcal{C}$ and $\sum_{j=0}^{n} \sum_{i=0}^{m_{j}} a_{i, j} x^{i} y^{j}$ is irreducible in $\mathcal{C}[x, y]$. When $n=1, \hat{y}$ is called a rational general solution of $F=0$.

For algebraic solutions of a differential equation $F=0$, we have the following lemma.

Lemma 2.4. Let $G(y) \in \mathcal{C}(x)[y]$ and irreducible in $\overline{\mathcal{C}}(x)[y]$ where $\overline{\mathcal{C}}$ is the algebraic closure of $\mathcal{C}$. If one solution of $G(y)=0$ is a solution of $F=0$, then every solution of $G(y)=0$ is the solution of $F=0$.

Proof. Since $G(y)$ is irreducible in $\overline{\mathcal{C}}(x)[y]$, every solution of $G(y)=0$ is a generic zero of $G(y)=0$. By Lemma $2.2, \operatorname{prem}(F, G)=0$. That is,

$$
S^{k} I^{l} F=P G^{\prime}+Q G
$$

where $S=\frac{\partial G}{\partial y}, I$ is the initial of $G$ and $k, l \in \mathbb{Z}$. Since every solution of $G(y)=0$ is a generic zero, it does not vanish $S$ or $I$. Hence every solution of $G(y)=0$ is a solution of $F=0$.

$$
\text { I }
$$

A general solution of $F=0$ is usually defined as a family of solutions with $o$ independent parameters in a loose sense where $o=\operatorname{ord}(F)$. The definition given by Ritt is more precise. Theorem 6 in section 12, chapter 2 in [13] tells us that Ritt's definition of general solutions is equivalent to the definition in the classical literature.

\subsection{A Criterion for existence of algebraic gen- eral solutions}

For non-negative integers $h, \alpha, k$, let $\mathcal{A}_{(h, \alpha ; k)}(y)$ be the following $(h+1) \times(\alpha+1)$ matrix:

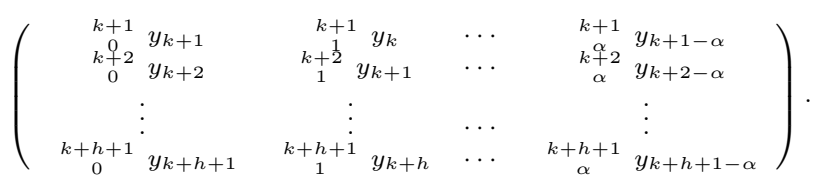

Let $\underline{\alpha}=\left(\alpha_{1}, \cdots, \alpha_{n}\right) \in \mathbb{Z}_{>0}^{n}, \alpha_{0} \in \mathbb{Z}_{\geq 0}$ where $\mathbb{Z}_{\geq 0}$ means the set of non-negative integers. Let $\mathcal{A}_{\left(\alpha_{0} ; \underline{\alpha}\right)}(y)$ be the $(h+$ 1) $\times(h+1)$ matrix

$$
\left(\mathcal{A}_{\left(h, \alpha_{1} ; \alpha_{0}\right)}(y)\left|\mathcal{A}_{\left(h, \alpha_{2} ; \alpha_{0}\right)}\left(y^{2}\right)\right| \cdots \mid \mathcal{A}_{\left(h, \alpha_{n} ; \alpha_{0}\right)}\left(y^{n}\right)\right)
$$

where $n+\alpha_{1}+\cdots+\alpha_{n}=h+1$. Let $\mathcal{D}_{\left(\alpha_{0} ; \underline{\alpha}\right)}$ be the determinant of $\mathcal{A}_{\left(\alpha_{0} ; \underline{\alpha}\right)}(y)$. Note that if $n=1, \mathcal{D}_{\left(\alpha_{0}, \underline{\alpha}\right)}$ is just equal to $\mathcal{D}_{n, m}$ in [9].

Lemma 2.5. An element $\bar{y}$ in the universal extension of $\mathbf{K}$ is a solution of $\mathcal{D}_{\left(\alpha_{0} ; \underline{\alpha}\right)}=0$ iff it satisfies equation (3) with $m_{i} \leq \alpha_{i}$ for $i=0, \cdots, n$. 
Proof. Assume that $\bar{y}$ satisfies the equation (3) with $m_{i} \leq \alpha_{i}$ where $i=0, \cdots, n$. Then we have

$$
\sum_{j=1}^{n} \sum_{i=0}^{m_{j}} a_{i, j}\left(x^{i} \bar{y}^{j}\right)^{\left(m_{0}+1\right)}=0
$$

where $\left(x^{i} \bar{y}^{j}\right)^{\left(m_{0}+1\right)}$ means the $\left(m_{0}+1\right)$-th derivative of $x^{i} \bar{y}^{j}$ with respect to $x$. Since $a_{i, j}$ are constants, $\left(x^{i} \bar{y}^{j}\right)^{\left(m_{0}+1\right)}$ $\left(i=0, \cdots, m_{j}, j=1, \cdots, n\right)$ are linearly dependent over $\mathcal{C}$. That is, the Wronskian determinant $W\left(\left(x^{i} \bar{y}^{j}\right)^{\left(m_{0}+1\right)}\right)$ for $\left(x^{i} \bar{y}^{j}\right)^{\left(m_{0}+1\right)}$ vanishes where $j=0, \cdots, n, i=0, \cdots, m_{j}$ [18]. Then $\bar{y}$ satisfies the equation (3) with $m_{i} \leq \alpha_{i}$ iff $W\left(\left(x^{i} \bar{y}^{j}\right)^{\left(m_{0}+1\right)}\right)=0$. By the computation process,

$$
W\left(\left(x^{i} \bar{y}^{j}\right)^{\left(m_{0}+1\right)}\right)=\mathcal{D}_{\left(\alpha_{0} ; \underline{\alpha}\right)}(\bar{y}) *\left|\operatorname{diag}\left(B_{0}, \cdots, B_{n}\right)\right|
$$

where $\operatorname{diag}\left(B_{0}, \cdots, B_{n}\right)$ is the diagonal matrix of $B_{i}$ and

$$
B_{i}=\left(\begin{array}{cccc}
1 & x & \cdots & x^{\alpha_{i}} \\
0 & 1 & \cdots & \alpha_{i} x^{\alpha_{i}-1} \\
\vdots & \vdots & \cdots & \vdots \\
0 & 0 & \cdots & \alpha_{i} !
\end{array}\right)
$$

for $i=0, \cdots, n$. Hence $W\left(\left(x^{i} \bar{y}^{j}\right)^{\left(m_{0}+1\right)}\right)=0 \Longleftrightarrow \mathcal{D}_{\left(\alpha_{0} ; \underline{\alpha}\right)}(\bar{y})=$ 0 .

$$
\text { I }
$$

By the above Lemma, we can prove the following criteria theorem easily.

THEOREM 2.6. Let $F$ be an irreducible differential polynomial. Then $F=0$ has an algebraic general solution $\hat{y}$ iff there exist $\underline{\alpha}=\left(\alpha_{1}, \cdots, \alpha_{n}\right) \in \mathbb{Z}_{\geq 0}^{n}, \alpha_{0} \in \mathbb{Z}_{\geq 0}$ such that $\operatorname{prem}\left(\mathcal{D}_{\left(\alpha_{0} ; \underline{\alpha}\right)}, F\right)=0$.

Proof. $(\Rightarrow)$ Let $\hat{y}$ be an algebraic general solution of $F=$ 0 which satisfies the equation (3). Let $\underline{\alpha}=\left(m_{1}, m_{2}, \cdots, m_{n}\right)$ and $\alpha_{0}=m_{0}$. Then from Lemmas 2.1, 2.2 and 2.5

$$
\mathcal{D}_{\left(\alpha_{0}, \underline{\alpha}\right)}(\hat{y})=0 \Rightarrow \mathcal{D}_{\left(\alpha_{0}, \underline{\alpha}\right)} \in \Sigma_{F} \Rightarrow \operatorname{prem}\left(\mathcal{D}_{\left(\alpha_{0}, \underline{\alpha}\right)}, F\right)=0 .
$$

$(\Leftarrow)$ By Lemma 2.1, $\operatorname{prem}\left(\mathcal{D}_{\left(\alpha_{0}, \alpha\right)}, F\right)=0$ which implies that $\mathcal{D}_{\left(\alpha_{0}, \underline{\alpha}\right)} \in \Sigma_{F}$. Then all the zeros of $\Sigma_{F}$ must satisfy the equation (3). In particular, the generic zero of $\Sigma_{F}$ satisfies the equation (3).

$$
\text { I }
$$

Given an algebraic differential equation $F=0$, if we know the degree bound of the equation (3) with respect to $x$ and $y$ which perhaps defines an algebraic general solution of $F=0$, then we can decide whether it has an algebraic general solution by computing $\operatorname{prem}\left(\mathcal{D}_{\left(\alpha_{0}, \underline{\alpha}\right)}, F\right)$ step by step. However for ODEs with order greater than one or with variate coefficients, we do not know this bound. Even for the case $y^{\prime}=\frac{P(x, y)}{Q(x, y)}$ where $P(x, y), Q(x, y) \in \mathbf{Q}[x, y]$, we have no effective method to get the bound $[3,16]$. In the following, for first order autonomous ODEs, we give a degree bound for its algebraic function solutions.

\section{DEGREE BOUND FOR FIRST ORDER AUTONOMOUS ODES}

In the following, we will always assume that $F=0$ is a first order autonomous ODE in $\mathbf{Q}\{y\}$ and irreducible in $\overline{\mathbf{Q}}\{y\}$ and $G(x, y) \in \overline{\mathbf{Q}}[x, y]$ which is irreducible. We say $G(x, y)$ is nontrivial if $\operatorname{deg}(G, x)>0$ and $\operatorname{deg}(G, y)>0$. From now on, we always assume that $G(x, y)$ is nontrivial. When we say that $G(x, y)=0$ is an algebraic solution of $F=0$, we mean that one of the algebraic functions $\hat{y}(x)$ defined by $G(x, \hat{y}(x))=0$ is a solution of $F=0$.

\subsection{Structure for algebraic general solutions}

It is a trivial fact that for an autonomous ODE, the solution set is invariant by a translation of the independent variable $x$. Moreover, we have the following fact.

LEMMA 3.1. Let $G(x, y)=0$ be an algebraic solution of $F=0$. Then $G(x+c, y)=0$ is an algebraic general solution of $F=0$, where $c$ is an arbitrary constant.

Proof. Assume that $\bar{y}(x)$ is a formal power series solution of $G(x, y)=0$. Then $\bar{y}(x+c)$ will be a solution of $G(x+c, y)=0$. Because $\bar{y}(x)$ is a solution of $F=0$, $\bar{y}(x+c)$ is still a solution of $F=0$. Hence $G(x+c, y)=0$ is an algebraic solution of $F=0$. For any $T \in \mathbf{K}\{y\}$ satisfying $T(\bar{y}(x+c))=0$, let $R=\operatorname{prem}(T, F)$. Then $R(\bar{y}(x+c))=0$. Suppose that $R \neq 0$. Since $F$ is irreducible and $\operatorname{deg}\left(R, y_{1}\right)<\operatorname{deg}\left(F, y_{1}\right)$, there are two differential polynomials $P, Q \in \mathbf{K}\{y\}$ such that $P F+Q R \in \mathbf{K}[y]$ and $P F+Q R \neq 0$. Thus $(P F+Q R)(\bar{y}(x+c))=0$. Because $\bar{y}(x+c) \notin \overline{\mathbf{Q}}$ and $c$ is an arbitrary constant which is transcendental over $\mathbf{K}$, we have $P F+Q R=0$, a contradiction. Hence $R=0$ which means that $T \in \Sigma_{F}$. So $\bar{y}(x+c)$ is a generic zero of $\Sigma_{F}$. Hence $G(x+c, y)=0$ is an algebraic general solution.

Lemma 3.1 reduces the problem of finding an algebraic general solution to the problem of finding a nontrivial algebraic solution. In what below, we will show how to find a nontrivial algebraic solution in $\mathbf{\mathbf { Q }}[x, y]$. First of all, we decide the degree of an algebraic solution.

\subsection{Degree bound of an algebraic solution}

Assume that $G(x, y)=0$ is an algebraic solution of differential equation $F=0$. In this subsection, we will give a bound for $\operatorname{deg}(G, x)$ and $\operatorname{deg}(G, y)$. First of all, we introduce some concepts on algebraic function field in one variable.

DEFINITION 3.2. $\overline{\mathbf{Q}}(x, \alpha)$ is called an algebraic function field in one variable, if $x$ is transcendental over $\overline{\mathbf{Q}}$ and $\alpha$ is algebraic over $\overline{\mathbf{Q}}(x)$ [11].

For an irreducible algebraic curve $G(x, y)=0$ where $G(x, y) \in$ $\overline{\mathbf{Q}}[x, y]$, it corresponds to a unique algebraic function field $\overline{\mathbf{Q}}(\alpha, \beta)$ under isomorphism where $\alpha, \beta$ satisfies $G(\alpha, \beta)=0$ and $\alpha$ or $\beta$ is transcendental over $\overline{\mathbf{Q}}$. It is well known that two algebraic curves with isomorphic function fields have the same genus.

\subsubsection{Parametrization of a curve}

Let $\mathbf{Q}((t))$ be the quotion field of the ring of formal power series $\mathbf{Q}[[t]]$. Let $G(x, y)$ be a nontrivial irreducible polynomial of $\overline{\mathbf{Q}}[x, y]$. If $x(t), y(t) \in \overline{\mathbf{Q}}((t))$ such that $G(x(t), y(t))=0$, 
we say that they are the coordinates of a parametrization provided $x(t)$ or $y(t)$ does not belong to $\mathbf{Q}$. There exist $x_{0}, y_{0} \in \overline{\mathbf{Q}}$, nonzero integers $q$ and $p$, and units $u(t), v(t)$ in $\overline{\mathbf{Q}}[[t]]$, such that

$$
\begin{aligned}
& x(t)-x_{0}=t^{q} u(t), \\
& y(t)-y_{0}=t^{p} v(t) .
\end{aligned}
$$

The center of the parametrization is the point $P \in \mathbb{P}^{1} \times \mathbb{P}^{1}$ defined accordingly the following cases: (a) If $q>0$ and $p>0$, then $P=\left(x_{0}, y_{0}\right)$; (b) If $q>0$ and $p<0$, then $P=\left(x_{0}, \infty\right)$; (c) If $q<0$ and $p>0$, then $P=\left(\infty, y_{0}\right)$; (d) If $q<0$ and $p<0$, then $P=(\infty, \infty)$. If $p<0$ (resp. $q<0)$ we agree to take $y_{0}=0$ (resp. $\left.x_{0}=0\right)$.

If there exists an integer $k \geq 2$ such that $x(t), y(t) \in \overline{\mathbf{Q}}\left(\left(t^{k}\right)\right)$, the parametrization will be called reducible, otherwise irreducible. If $\bar{t} \in \overline{\mathbf{Q}}[[t]]$ with order with respect to $t$ greater than zero, then $x(\bar{t}), y(\bar{t})$ is another parametrization with the same center. If the order of $\bar{t}$ is equal to one, the two parametrizations will be said to be equivalent. An equivalence class of irreducible parametrizations will be called a place $B$ of the curve $G=0$ with center the center of one of its parametrizations. Two equivalent parametrizations have the same integers $q$ and $p$ as defined above. Then given a place $B$, we define nonzero integers $\nu_{x}(B)$ and $\nu_{y}(B)$ as the integers $q$ and $p$ of any of its parametrizations.

Let $g$ be the genus of $G(x, y)=0$ and $n=\operatorname{deg}(G, y)$. By the Riemann-Hurwitz formula [15] we have that

$$
g=1-n+\frac{1}{2} \sum_{B}\left(\left|\nu_{x}(B)\right|-1\right)
$$

where $B$ runs over all places of the curve $G=0$.

For each place $B$ with center $(\alpha, \beta)$ it corresponds exactly $q_{B}$ fractional power series $y\left(x^{1 / q}\right)$ which are solutions of $G(x, y(x))=0$. Let $\alpha \in \overline{\mathbf{Q}} \cup\{\infty\}$. Hence, by the Puiseux theorem we have that

$$
\sum_{B}\left|\nu_{x}(B)\right|=\operatorname{deg}(G, y)
$$

where the sum run over all places $B$ of the curve $G=0$ with center $(\alpha, \beta)$.

LEMMA 3.3. Let $G(x, y)$ be a nontrivial irreducible polynomial of $\overline{\mathbf{Q}}[x, y]$. Let $(x(t), y(t))$ be the coordinates of a parametrization $G=0$. Then, for any nonzero constant $c \in$ $\overline{\mathbf{Q}},(x(t)+c, y(t))$ are not the coordinates of a parametrization of $G=0$.

Proof. Assume that $(x(t)+c, y(t))$ are the coordinates of a parametrization of $G=0$. We may assume that $y(t)=$ $t^{p}$ by substituting $y-y_{0}$ in $G$ and taken an appropriate equivalent parametrization (see Theorem 2.2 of chapter IV in [26]). Then we have that $G\left(x\left(y^{1 / p}\right), y\right)=0=G\left(x\left(y^{1 / p}\right)+\right.$ $c, y)$. Let us consider $H(x, y)=G(x+c, y)$. Then $G$ and $H$ are irreducible polynomials in $\overline{\mathbf{Q}}[x, y]$ of the same degree and having a common root, hence there exists $\lambda \in \overline{\mathbf{Q}}$ such that $G=\lambda H$. This implies that $\operatorname{deg}(G, x)=0$, in contradiction with the nontriviality of $G$.
Now we are ready to give the degree bound of the algebraic solution of $F=0$. First, we could determine the degree $\operatorname{deg}(G, x)$ exactly from the degree of $F$.

TheOrem 3.4. Let $G(x, y) \in \overline{\mathbf{Q}}[x, y]$ be irreducible and $G(x, y)=0$ is an algebraic solution of $F=0$. Then we have

$$
\operatorname{deg}(G, x)=\operatorname{deg}\left(F, y_{1}\right) .
$$

Proof. Assume that $\operatorname{deg}(G, x)=s$ and $\operatorname{deg}\left(F, y_{1}\right)=d$. Let us write

$$
\begin{aligned}
G(x, y) & =A_{0}(y)+A_{1}(y) x+\cdots+A_{s}(y) x^{s}, \\
F & =F_{0}(y)+F_{1}(y) y_{1}+\cdots+F_{d}(y) y_{1}^{d}
\end{aligned}
$$

where $A_{i}(y), F_{j}(y) \in \overline{\mathbf{Q}}[y]$. We use $\operatorname{Res}(A, B, z)$ to denote the Sylvester-resultant of $A$ and $B$ with respect to $z$ and $\mathcal{Z}$ stands for "the zero set of". Let $S=\mathcal{Z}\left(A_{s}(y)\right) \cup \mathcal{Z}\left(F_{d}(y)\right) \cup$ $\mathcal{Z}\left(\operatorname{Res}\left(G, \frac{\partial G}{\partial x}, x\right)\right) \cup \mathcal{Z}\left(\operatorname{Res}\left(G, \frac{\partial G}{\partial y}, x\right)\right) \cup \mathcal{Z}\left(\operatorname{Res}\left(F, \frac{\partial F}{\partial y_{1}}, y_{1}\right)\right)$. Then $S$ is a finite set. Hence we can choose a $c \in \overline{\mathbf{Q}}$ such that $c \notin S$. Then we have the following results.

- The set $\{z \in \overline{\mathbf{Q}} \mid F(c, z)=0\}=\left\{z_{1}, z_{2}, \cdots, z_{d}\right\}$ has exactly $d$ elements;

- The set $\{x \in \overline{\mathbf{Q}} \mid G(x, c)=0\}=\left\{x_{1}, x_{2}, \cdots, x_{s}\right\}$ has exactly $s$ elements;

- Since $\frac{\partial G}{\partial y}\left(x_{i}, c\right) \neq 0$, there exists a unique formal power series $y_{i}(x)=c+g_{i, 1}\left(x-x_{i}\right)+g_{i, 2}\left(x-x_{i}\right)^{2}+\cdots$ such that $G\left(x, y_{i}(x)\right)=0$ for each $i=1, \cdots, s$.

From Lemma 2.4, $y_{i}(x)$ is a solution of $F=0$. That is, $F\left(y_{i}(x), y_{i}^{\prime}(x)\right)=0$ which implies that $F\left(c, g_{i, 1}\right)=0$. Suppose that $s>d$. Then there exist at least two of $g_{i, 1}$ which are equal to each other. Without lost of generalization, assume that we have $g_{1,1}=g_{2,1}=c_{1}$. Since $\frac{\partial F}{\partial y_{1}}\left(c, c_{1}\right) \neq 0$, there exist only one solution $y(x)$ of $F\left(y, y^{\prime}\right)=0$ such that $y(0)=c$ and $y^{\prime}(0)=c_{1}$. Hence $y_{1}(x)=y_{2}\left(x+x_{2}-x_{1}\right)=$ $y\left(x-x_{1}\right)$. Hence $\left(x, y_{1}(x)\right)$ and $\left(x+x_{2}-x_{1}, y_{1}(x)\right)$ are two coordinates of a parametrizations of $G=0$. This is a contradiction by the above lemma. Hence $s \leq d$.

Let $G^{\prime}=y_{1} \frac{\partial G}{\partial y}+\frac{\partial G}{\partial x}$ and $H\left(y, y_{1}\right)=\operatorname{Res}\left(G, G^{\prime}, x\right)$. Then $H\left(y, y_{1}\right)=y_{1}^{s} \operatorname{Res}\left(G, \frac{\partial G}{\partial y}, x\right)+$ terms of lower order in $y_{1}$.

Since $\operatorname{Res}\left(G, \frac{\partial G}{\partial y}, x\right) \neq 0$, we have $\operatorname{deg}\left(H, y_{1}\right)=s$. Assume that $\bar{y}(x)$ is a solution of $G(x, y)=0$. Then we have $H\left(\bar{y}(x), \bar{y}^{\prime}(x)\right)=F\left(\bar{y}(x), \bar{y}^{\prime}(x)\right)=0$. Because $F$ is irreducible, we have that $\operatorname{deg}\left(H, y_{1}\right) \geq \operatorname{deg}\left(F, y_{1}\right)$. In the other word, $s \geq d$.

I

Since $F$ is first order and autonomous, we can regard $F=0$ as an algebraic curve and we will use $F\left(y, y_{1}\right)$ to denote $F$.

Lemma 3.5. Assume that $G(x, y)=0$ is an algebraic solution of $F=0$. Then the genus of $G(x, y)=0$ equals to that of $F\left(y, y_{1}\right)=0$. 
Proof. Let $\alpha$ satisfy $G(x, \alpha)=0$. It is clear that $\alpha$ is transcendental over $\overline{\mathbf{Q}}$. Then $\overline{\mathbf{Q}}(x, \alpha)$ and $\overline{\mathbf{Q}}\left(\alpha, \alpha^{\prime}\right)$ are the algebraic function fields of $G(x, y)=0$ and $F\left(y, y_{1}\right)=0$ respectively. We only need to prove $\overline{\mathbf{Q}}(x, \alpha)=\overline{\mathbf{Q}}\left(\alpha, \alpha^{\prime}\right)$. From Theorem 3.4, we have $[\overline{\mathbf{Q}}(x, \alpha): \overline{\mathbf{Q}}(\alpha)]=\left[\overline{\mathbf{Q}}\left(\alpha, \alpha^{\prime}\right)\right.$ : $\overline{\mathbf{Q}}(\alpha)]$. Since $G(x, \alpha)=0, \alpha^{\prime}=-\frac{\partial G}{\partial x}(x, \alpha) / \frac{\partial G}{\partial y}(x, \alpha)$. which implies that $\alpha^{\prime} \in \overline{\mathbf{Q}}(x, \alpha)$. Hence $\overline{\mathbf{Q}}(x, \alpha)=\overline{\mathbf{Q}}\left(\alpha, \alpha^{\prime}\right)$.

For convenience, we consider a new differential equation

$$
\bar{F}\left(x_{1}, y\right)=x_{1}^{\operatorname{deg}\left(F, y_{1}\right)} F\left(y, \frac{1}{x_{1}}\right)=0
$$

where $x_{1}=\frac{d x}{d y}=\frac{1}{y_{1}} . \quad \bar{F}$ is irreducible in $\overline{\mathbf{Q}}\left[x_{1}, y\right]$ and $\operatorname{deg}(\bar{F}, y)=\operatorname{deg}(F, y), \operatorname{deg}\left(\bar{F}, x_{1}\right)=\operatorname{deg}\left(F, y_{1}\right)$. If $G(x, y)=$ 0 is an algebraic solution of $F=0$, then we will prove that it is also an algebraic solution of $\bar{F}=0$.

Lemma 3.6. Let $\bar{F}$ be defined as in (6) and $G(x, y)=0$ an algebraic solution of $F=0$. Then $G(x, y)=0$ also defines an algebraic function (in $y$ ) solution of $\bar{F}\left(x_{1}, y\right)=0$.

Proof. From the proof of Theorem 3.4, we know that

$$
\operatorname{Res}\left(G, G^{\prime}, x\right)=A(y) F\left(y, y_{1}\right)
$$

where $G^{\prime}=y_{1} \frac{\partial G}{\partial y}+\frac{\partial G}{\partial x}$. In the other word, there exist two polynomials $P, Q \in \overline{\mathbf{Q}}\left[x, y, y_{1}\right]$ such that $P G+Q G^{\prime}=$ $A(y) F\left(y, y_{1}\right)$. Substituting $y_{1}$ by $\frac{1}{x_{1}}$ and multiplying some power of $x_{1}$, we have

$$
\bar{P} G+\bar{Q}\left(\frac{\partial G}{\partial y}+x_{1} \frac{\partial G}{\partial x}\right)=x_{1}^{k} A(y) \bar{F}\left(x_{1}, y\right)
$$

where $\bar{P}, \bar{Q} \in \overline{\mathbf{Q}}\left[x, y, x_{1}\right]$ and $k \in \mathbb{Z}_{\geq 0}$. Suppose that $\beta$ satisfies $G(\beta, y)=0$. Substituting $x$ by $\beta$ and $x_{1}$ by $\beta^{\prime}$ in $(7)$ where $\beta^{\prime}=\frac{d \dot{\beta}}{d y}$, we have that $\bar{F}\left(\beta^{\prime}, y\right)=0$. Hence $G(x, y)=0$ is an algebraic solution of $\bar{F}=0$.

Lemma 3.7. Let $(x(t), y(t))$ be an irreducible parametrization of $G=0$. Then $\left(\frac{x^{\prime}(t)}{y^{\prime}(t)}, y(t)\right)$ is an irreducible parametrization of $\bar{F}\left(x_{1}, y\right)=0$.

Proof. Let us denote $x_{1}(t)=\frac{x^{\prime}(t)}{y^{\prime}(t)}$ where ' means the derivative with respect to $t$. Since $x_{1}(t)=\frac{d x(t)}{d y(t)}$, we have $\bar{F}\left(x_{1}(t), y(t)\right)=0$. Assume that $\left(x_{1}(t), y(t)\right)$ is a reducible parametrization. Let $k \geq 2$, such that $x_{1}(t), y(t) \in \overline{\mathbf{Q}}\left(\left(t^{k}\right)\right)$. Then $x_{1}(t) y^{\prime}(t)=\sum_{j \geq j_{0}} c_{j} t^{k j-1}$. Since $x^{\prime}(t)=x_{1}(t) y^{\prime}(t)$, then we have that $c_{0}=0$ and $x(t)=c+\sum_{j \geq j_{0}} \frac{c_{j} t^{k j}}{k j}$, for some constant $c$. Hence we get a contradiction because $x(t), y(t) \in \overline{\mathbf{Q}}\left(\left(t^{k}\right)\right)$.

Theorem 3.8. Assume that $G(x, y)=0$ is a nontrivial algebraic solution of $F=0$. Then we have that

$$
\operatorname{deg}(G, y) \leq \operatorname{deg}(F, y)+\operatorname{deg}\left(F, y_{1}\right) .
$$

Proof. Let $\bar{F}$ be as in (6). Let $g_{G}$ and $g_{\bar{F}}$ be the genus of $G(x, y)=0$ and $\bar{F}\left(x_{1}, y\right)=0$ respectively. Let $B$ be a place of $G=0$ with center $P=(\alpha, \beta)$. Let $(x(t), y(t))$ be an irreducible parametrization of $B$. Let us denote by $\tilde{B}$ the place of the algebraic curve $\bar{F}\left(x_{1}, y\right)=0$ given by the irreducible parametrization $\left(x_{1}(t), y(t)\right)$, where $x_{1}(t)=$ $x^{\prime}(t) / y^{\prime}(t)$. Let $\tilde{P}=(\tilde{\alpha}, \tilde{\beta})$ be the center of $\tilde{B}$. It is obvious that $\nu_{y}(B)=\nu_{y}(\tilde{B})$ and $\beta=\tilde{\beta}$. If $\nu_{x}(B) \neq \nu_{y}(B)$ then we have that $\nu_{x_{1}}(\tilde{B})=\nu_{x}(B)-\nu_{y}(B)$. Hence, if $\nu_{x}(B)>$ $\nu_{y}(B)$, then $\tilde{\alpha}=0$; if $\nu_{x}(B)<\nu_{y}(B)$, then $\tilde{\alpha}=\infty$; if $\nu_{x}(B)=\nu_{y}(B)$, then $\tilde{\alpha} \in \overline{\mathbf{Q}}$.

The map that sends each place $B$ of $G=0$ to the place $\tilde{B}$ is injective. Let $B$ and $B^{\prime}$ be two places of $G=0$ such that $\tilde{B}=\widetilde{B^{\prime}}$. Let $(x(t), y(t))$ and $(z(t), v(t))$ be the parametrizations of $B$ and $B^{\prime}$ respectively. We may assume that $y(t)=y_{0}+t^{p}$ and $v(t)=v_{0}+t^{p^{\prime}}$. Since $\tilde{B}=\widetilde{B^{\prime}}$ we have that $p=p^{\prime}, y(t)=v(t)$ and $x^{\prime}(t)=z^{\prime}(t)$. Hence $z(t)=x(t)+c$, for some constant $c$. By lemma 3.3 we have that $c=0$, so $B=B^{\prime}$.

By Riemann-Hurwitz formula we have that

$$
2\left(g_{G}+\operatorname{deg}(G, y)-1\right)=\sum_{B}\left(\left|\nu_{x}(B)\right|-1\right),
$$

where $B$ runs over all places of $G=0$.

We will split the right hand side of the above equation in four cases: We say that $B \in(1)$ if $\nu_{x}(B)>0$ and $\nu_{y}(B)>0$; that $B \in(2)$ if $\nu_{x}(B)>0$ and $\nu_{y}(B)<0 ; B \in(3)$ if $\nu_{x}(B)<0$ and $\nu_{y}(B)>0$; and that $B \in(4)$ if $\nu_{x}(B)<0$ and $\nu_{y}(B)<0$. Moreover, we say that $B \in(1)^{\prime}$ if $B \in(1)$ and $\nu_{x}(B)>\nu_{y}(B)$; and we say that $B \in(4)^{\prime}$ if $B \in$ (4) and $\nu_{x}(B)<\nu_{y}(B)$. In the following equations $B_{x}, B_{y}$ $\tilde{B}_{x_{1}}$ and $\tilde{B}_{y}$ will stand for $\nu_{x}(B), \nu_{y}(B), \nu_{x_{1}}(\tilde{B})$ and $\nu_{y}(\tilde{B})$ respectively.

For $k=1$ and $k=4$, we have that

$$
\sum_{B \in(k)}\left(\left|B_{x}\right|-1\right) \leq \sum_{B \in(k)^{\prime}}\left|\tilde{B}_{x_{1}}\right|+\sum_{B \in(k)}\left(\left|B_{y}\right|-1\right) .
$$

For $k=2$ and $k=3$, we have that

$$
\sum_{B \in(k)}\left(\left|B_{x}\right|-1\right) \leq \sum_{B \in(k)}\left|\tilde{B}_{x_{1}}\right| .
$$

If $B \in(1)^{\prime} \cup(2)$, then the center of $\tilde{B}$ is over $x_{1}=0$. If $B \in(3) \cup(4)^{\prime}$, then the center of $\tilde{B}$ is over $x_{1}=\infty$. Hence, using formula (5), we have that

$$
\sum_{B \in(1)^{\prime},(2),(3),(4)^{\prime}}\left|\tilde{B}_{x_{1}}\right| \leq 2 \operatorname{deg}(\bar{F}, y) .
$$

By the Riemann-Hurwitz formula, we have that

$$
\sum_{B \in(1),(4)}\left(\left|\tilde{B}_{y}\right|-1\right) \leq 2\left(g_{\bar{F}}+\operatorname{deg}\left(\bar{F}, x_{1}\right)-1\right) .
$$

We remark that in equations $(11,12)$ we have used the fact that the map $B \mapsto \tilde{B}$ between the places of $G=0$ and places of $\bar{F}=0$ is injective. By equations ((8)-(12)), we have that $2\left(g_{G}+\operatorname{deg}(G, y)-1\right) \leq 2\left(g_{\bar{F}}+\operatorname{deg}\left(\bar{F}, x_{1}\right)+\operatorname{deg}(\bar{F}, y)-1\right)$. Using the above equation, and the facts that $\operatorname{deg}\left(\bar{F}, x_{1}\right)=$ $\operatorname{deg}\left(F, y_{1}\right), \operatorname{deg}(\bar{F}, y)=\operatorname{deg}(F, y)$ and that $g_{G}=g_{\bar{F}}$, gives the required inequality.
I 
The following example shows that the degree bound given in Theorem 3.8 is optimized.

EXAmple 3.9. Assume that $n>m>0$ and $(n, m)=1$. Let $G(x, y)=y^{n}-x^{m}$ which is irreducible. We have that $G(x, y)=0$ is an algebraic solution of $F=y^{n-m} y_{1}^{m}-$ $\left(\frac{m}{n}\right)^{m}=0$. In this case, we have that $\operatorname{deg}(G, y)=\operatorname{deg}(F, y)+$ $\operatorname{deg}\left(F, y_{1}\right)$.

\section{A POLYNOMIAL-TIME ALGORITHM}

The simple degree bounds given in the preceding section allow us to give a polynomial-time algorithm to compute algebraic function solutions of a first order autonomous ODE.

\subsection{Algebraic approximant}

Algebraic approximant is a special type of Hermite-Padé approximant. It uses an algebraic function to approximate a given function.

DeFinition 4.1. Let $G(x, y)$ be an irreducible polynomial in $\overline{\mathbf{Q}}[x, y]$. An algebraic function $\bar{y}(x)$ satisfying $G(x, \bar{y}(x))=$ 0 is called an algebraic approximant to a function $f(x)$ if

$$
G(x, f(x))=O\left(x^{(m+1)(n+1)-1}\right)
$$

where $m=\operatorname{deg}(G, x)$ and $n=\operatorname{deg}(G, y)$.

More generally, we will find $G(x, y)$ such that

$$
G(x, f(x))=O\left(x^{N+1}\right)
$$

where $N$ is a positive integer. We can get the coefficients of $G(x, y)$ with respect to $x$ and $y$ by solving linear equations. Let $G(x, y)=\sum_{j=0}^{n} \sum_{i=0}^{m} b_{i, j} x^{i} y^{j}$ and $f(x)=a_{0}+a_{1} x+$ $\cdots+a_{N} x^{N}+O\left(x^{N+1}\right)$. Let

$$
M_{0}=\left(\begin{array}{c}
I_{(m+1) \times(m+1)} \\
0_{(N-m) \times(m+1)}
\end{array}\right)
$$

where $I_{(m+1) \times(m+1)}$ is an $m+1$ unit square matrix, $0_{(N-m) \times(m+1)}$ is an $(N-m) \times(m+1)$ zero matrix. Let $M_{i}=T M^{i} * M_{0}$ for $i=1, \cdots, n$ where

$$
T M=\left(\begin{array}{ccccc}
a_{0} & 0 & 0 & \cdots & 0 \\
a_{1} & a_{0} & 0 & \cdots & 0 \\
a_{2} & a_{1} & a_{0} & \cdots & 0 \\
\vdots & \vdots & \vdots & \vdots & \vdots \\
a_{N} & a_{N-1} & a_{N-2} & \cdots & a_{0}
\end{array}\right)
$$

and $a_{i}$ are the coefficients of $f(x)$. Then by the computation process, we can write (13) as the matrix form

$$
\left(M_{0}\left|M_{2}\right| \cdots \mid M_{n}\right) \quad\left(\begin{array}{c}
B_{0} \\
B_{1} \\
\vdots \\
B_{n}
\end{array}\right)=0, B_{i}=\left(\begin{array}{c}
b_{0, i} \\
b_{1, i} \\
\vdots \\
b_{m, i}
\end{array}\right)
$$

for $i=1, \cdots, n$.

Let $\bar{y}(x)=a_{0}+a_{1} x+\cdots$ be a formal power series. When we say $\varphi(x)$ is the first $N+1$ terms of $\bar{y}(x)$, we mean that $\varphi(x)=a_{0}+a_{1} x+\cdots+a_{N} x^{N}$. The following lemma will be used in our algorithm.
LEMMa 4.2. Let $\bar{y}(x)$ be a formal power series such that $G(x, \bar{y}(x))=0$. Assume that $m=\operatorname{deg}(G, x)$ and $n=$ $\operatorname{deg}(G, y)$. Let $\varphi(x)$ be the first $2 m n+1$ terms of $\bar{y}(x)$. If $Q_{0}(x), Q_{1}(x), \cdots, Q_{n}(x) \in \overline{\mathbf{Q}}[x]$ such that

$$
Q_{0}(x)+Q_{1}(x) \varphi(x)+\cdots+Q_{n}(x) \varphi(x)^{n}=O\left(x^{2 m n+1}\right)
$$

where $\operatorname{deg}\left(Q_{i}(x), x\right) \leq m$ and not all of them are zero. Then

$$
G(x, y)=\lambda\left(Q_{0}(x)+Q_{1}(x) y+\cdots+Q_{n}(x) y^{n}\right)
$$

where $\lambda \in \overline{\mathbf{Q}}$ does not equal to zero.

Proof. Let $Q(x, y)=Q_{0}(x)+Q_{1}(x) y+\cdots+Q_{n}(x) y^{n}$. There exist $S, T \in \overline{\mathbf{Q}}[x, y]$ such that

$$
S G(x, y)+T Q(x, y)=\operatorname{Res}(G, Q, y)
$$

where $\operatorname{deg}(S, y)<n$ and $\operatorname{deg}(T, y)<n$. If $Q(x, \bar{y}(x))=$ 0 , then (17) is true. Assume that $Q(x, \bar{y}(x)) \neq 0$ and $\operatorname{Res}(G, Q, y) \neq 0$. Then it is not difficult to know that $\operatorname{deg}(\operatorname{Res}(G, Q, y), x) \leq 2 m n$. However, substituting $\bar{y}(x)$ to the left side of (18), the left side will become a series with order greater than $2 m n$, a contradiction. Hence $\operatorname{Res}(G, Q, y)=$ 0 which implies $(17)$ is true, because $G(x, y)$ is irreducible.

\subsection{An algorithm to compute algebraic solu- tions}

First, we give an algorithm to compute the first $N+1$ terms of a formal power series solution of $F=0$ for a given positive integer $N$. Regarding $F=0$ as an algebraic curve, find a point $\left(z_{0}, z_{1}\right)$ on it such that it does not vanish the separant $S\left(y, y_{1}\right)$ of $F\left(y, y_{1}\right)$. Then we can compute $y_{i}=z_{i}$ step by step from (1). Then $\bar{y}(x)=z_{0}+z_{1} x+\frac{z_{2}}{2 !} x^{2}+\cdots$ is a formal power series solution of $F=0$. Moreover, if $z_{1} \neq 0$, then $\bar{y}(x) \notin \overline{\mathbf{Q}}$.

Algorithm 4.3. Input: $F=0$ and a positive integer $N$. Output: the first $N+1$ terms of a formal power series solution of $F=0$ which is not in $\overline{\mathbf{Q}}$.

1. Find a point $\left(z_{0}, z_{1}\right) \in \overline{\mathbf{Q}}^{2}$ on $F\left(y, y_{1}\right)=0$ such that $S\left(y, y_{1}\right) \neq 0$ and $y_{1} \neq 0$.

2. $i:=2$ and $\varphi(x):=z_{0}+z_{1} x$.

3. while $i \leq N$ do

(a) Substitute $y$ by $\varphi(x)$ and $y_{1}$ by $\varphi^{\prime}(x)$ in $F\left(y, y_{1}\right)$.

(b) $c:=$ the coefficient of $x^{i-1}$ in $F\left(\varphi(x), \varphi^{\prime}(x)\right)$.

(c) $z_{i}:=-\frac{(i-1) ! c}{S\left(z_{0}, z_{1}\right)}$ and $\varphi(x):=\varphi(x)+\frac{z_{i} x^{i}}{i !}$.

(d) $i:=i+1$.

4. $\operatorname{Return}(\varphi(x))$.

The correctness of the algorithm comes from the following facts. Let $\bar{y}(x)$ be a formal power series solution of $F=0$. Then by (1),

$\left(F\left(\bar{y}(x), \bar{y}_{1}(x)\right)\right)^{(i-1)}=S \bar{y}_{i}(x)+R\left(\bar{y}(x), \cdots, \bar{y}_{i-1}(x)\right)=0$.

Since $\left.\bar{y}_{k}(x)\right|_{x=0}=z_{k}$ for $k=1,2, \cdots$, we have that

$$
S\left(z_{0}, z_{1}\right) z_{i}+R\left(z_{0}, \cdots, z_{i-1}\right)=0 .
$$


Now assume that $\varphi(x)=z_{0}+z_{1} x+\cdots+\frac{z_{i-1}}{(i-1) !} x^{i-1}$. Then

$$
\left(F\left(\varphi(x), \varphi^{\prime}(x)\right)\right)^{(i-1)}=R\left(\varphi(x), \cdots, \varphi^{(i-1)}(x)\right) .
$$

Since $\left.\varphi^{(k)}(x)\right|_{x=0}=z_{k}$ for $k=1, \cdots, i-1$, we have that

$$
R\left(z_{0}, \cdots, z_{i-1}\right)=\left.\left(F\left(\varphi(x), \varphi^{\prime}(x)\right)\right)^{(i-1)}\right|_{x=0}
$$

which equals to $(i-1)$ ! times the coefficient of $x^{i-1}$ in $F\left(\varphi(x), \varphi^{\prime}(x)\right)$. Let $T=\operatorname{tdeg}(F)$, the total degree of $F$. Theorem 9 given in ([9]) shows that there is at most $T^{2}$ points on $F\left(y, y_{1}\right)=0$ which satisfy $S\left(y, y_{1}\right)=0$ or $y_{1}=0$.

The complexity of Algorithm 4.3 is polynomial in terms of the number of multiplications in $\mathbf{Q}$ needed in the algorithm. In Step 1, as proved in [9], we can find a point $\left(z_{0}, z_{1}\right)$ as follows. We may take $y$ to be an integer $z_{0}$ and let $L\left(y_{1}\right)$ be a monic irreducible factor of $F\left(z_{0}, y_{1}\right) \in \mathbf{Q}\left[y_{1}\right]$. We may take $z_{1}$ to be a root of $L\left(z_{1}\right)=0$. Then all the procedures will be executed over the number field $\mathbf{Q}\left(z_{1}\right)$. Let $D=$ $\operatorname{deg}\left(L\left(y_{1}\right)\right) \leq T=\operatorname{tdeg}(F)$. Then any element of $\mathbf{Q}\left(z_{1}\right)$ can be represented as a polynomial in $z_{1}$ with degree $\leq T-1$. Let $\beta, \gamma \in \mathbf{Q}\left(z_{1}\right)$. Then there exist $P(z), Q(z) \in \mathbf{Q}[z]$ such that $\beta=P(\alpha), \gamma=Q(\alpha)$ where $\operatorname{deg}(P) \leq T-1, \operatorname{deg}(Q) \leq$ $T-1$. To compute $\phi=\beta * \gamma$, we need to compute $\phi=$ $\operatorname{prem}(P Q, L)$. Therefore, a multiplication of two elements in $\mathbf{Q}\left(z_{1}\right)$ needs $O\left(T^{2}\right)$ multiplications of rational numbers. In Step 3, the computation of $\left(a_{0}+a_{1} x+\cdots+a_{N} x^{N}\right)^{T}$ needs at most $O\left(N^{2} T^{4}\right)$ multiplications in $\mathbf{Q}\left(z_{1}\right)$, and hence at most $O\left(T^{2} \cdot N^{2} T^{4}\right)=O\left(N^{2} T^{6}\right)$ multiplications in $\mathbf{Q}$.

Now we can give the algorithm to compute an algebraic solution of $F=0$.

Algorithm 4.4. Input: $F=0$. Output: an algebraic solution of $F=0$ if it exists.

1. $d:=\operatorname{deg}\left(F, y_{1}\right)$ and $e:=\operatorname{deg}(F, y)$.

2. $k:=1$

while $k \leq d+e$ do

(a) Compute the first $2 d k+1$ terms $\varphi(x)$ of a formal power series solution of $F=0$ by Algoritm 4.3.

(b) $a_{i}:=$ the coefficient of $x^{i}$ in $\varphi(x)$ for $i=0, \cdots, 2 d k$.

(c) In (14) and (15), let $m=d, n=k$ and $N=2 d k$. We construct the linear equations (16).

(d) If (16) has no nonzero solution or the dimension of the solution space of (16) is great than one, then go to Step (h).

(e) Otherwise, choose one of nonzero solutions $\bar{b}_{i, j}$ where $i=0, \cdots, d$ and $j=0, \cdots, k$.

(f) $G(x, y):=\sum_{j=0}^{k} \sum_{i=0}^{d} \bar{b}_{i, j} x^{i} y^{j}$.

(g) Let $R=\operatorname{prem}(F, G)$.

If $R=0$, then $\operatorname{return}(G(x, y)=0)$.

(h) $k:=k+1$.

3. If the algorithm does not return $G(x, y)=0$ in Step $2, F=0$ has no algebraic solution and the algorithm terminates.
From Theorem 2.6 and Lemma 2.5, we know that if $F=0$ has a nontrivial algebraic solution, then every formal power series solution is algebraic. From Lemma 4.2, we only need to compute the first $2 d k+1$ terms of a nontrivial formal power series solution to construct the algebraic approximant. From Theorems 3.4, 3.8, if $F=0$ has an algebraic solution $G(x, y)=0$, then there is a $k$ which satisfies that $k \geq 1$ and $k \leq d+e$ such that $\operatorname{deg}(G, x)=d$ and $\operatorname{deg}(G, y)=k$. In (14) and (15), let $m=d, n=k$ and $N=2 d k$. We construct the linear equations (16). From Lemma 4.2 again, the dimension of the solution space of (16) equals to one. At last, by Lemma $2.2, G(x, y)=0$ is an algebraic solution if $\operatorname{prem}(F, G)=0$.

The complexity of Algorithm 4.4 is polynomial in $T$ where $T=\operatorname{tdeg}(F)$. In Step 2(a), the complexity is polynomial. In Step 2(c), we need only to compute $T M^{2 T} * M_{0}$ which needs $O\left(T^{8}\right)$, because $T M$ is an $l \times l$ matrix with $l \leq 2 T^{2}+1$ and $M_{0}$ is a $p \times q$ matrix with $p \leq 2 T^{2}+1, q \leq T+1$. (Note that in the worst case, we have to do the operations over $\mathbf{Q}\left(z_{1}\right)$. Hence the complexity has to increase by $O\left(T^{2}\right)$.) In Step 2(d), we need only to solve at most $4 T^{2}+1$ linear equations with at most $2 T^{2}+3 T+1$ variables. Hence its complexity is polynomial. In Step $2(\mathrm{~g})$, for deciding whether $\operatorname{prem}(F, G)=0$, we compute $R_{1}=\operatorname{prem}\left(F, G^{\prime}\right)$ first. Since $R_{1}=\left(\frac{\partial G}{\partial y}\right)^{k} F\left(y,-\frac{\partial G}{\partial x} / \frac{\partial G}{\partial y}\right)$ where $k \leq T$, we can compute it in $O\left(T^{12}\right)$ and have that $\operatorname{deg}\left(R_{1}, x\right) \leq 2 T^{2}$ and $\operatorname{deg}\left(R_{1}, y\right) \leq$ $4 T^{2}+T$. Then we compute the $\operatorname{GCD}\left(R_{1}, G\right)$ which can be computed in $O\left(T^{10}\right)([25], \mathrm{p} 152)$. If $\operatorname{GCD}\left(R_{1}, G\right)=G$, then $\operatorname{prem}(F, G)=0$; otherwise $\operatorname{prem}(F, G) \neq 0$. The number of the circulation in Step 2 is at most $2 T$. Hence the complexity of Step 2 is also polynomial.

We have implemented the algorithm in Maple when $z_{0}$ and $z_{1}$ in Step 1 of Algorithm 4.3 are rational numbers.

\section{Example 4.5. Consider}

$F=\left(y^{6}+2 y+1\right) y_{1}^{3}-\left(12 y^{5}+9 y^{4}-1\right) y_{1}^{2}+27 y^{8}+54 y^{7}+27 y^{6}+4 y^{3}$.

1. Let $d=3$ and $e=8$.

2. $(1,-2)$ is a point on $F\left(y, y_{1}\right)=0$ which satisfies the assumption in Step 1 in Algorithm 4.3.

3. For the case $k=1$, we get a $G(x, y)=0$ which is not the solution of $F=0$. For saving the space, here we only give the process in the case $k=2$.

4. The first 13 terms of the formal power series solution of $F=0$ is

$$
\begin{aligned}
\varphi(x) & =1-2 x+\frac{5}{2} x^{2}-\frac{9}{4} x^{3}+\frac{1}{2} x^{4}+\frac{5}{4} x^{5}-\frac{41}{32} x^{6}-\frac{65}{64} x^{7} \\
& +\frac{363}{128} x^{8}-\frac{111}{256} x^{9}-\frac{2545}{512} x^{10}+\frac{5141}{1024} x^{11}+\frac{5891}{1024} x^{12} .
\end{aligned}
$$

5. Let $m=3, n=2$ and $N=12$. We construct the linear equations (16). Solving it, we get a nonzero solution

$$
(-1,1,0,0,0,3,-3,1,1,0,0,0) \text {. }
$$

6. Let $G(x, y)=-1+x+3 x y-3 x^{2} y+x^{3} y+y^{2}$.

7. $\operatorname{prem}(F, G)=0$. Hence $G(x, y)=-1+x+3 x y-$ $3 x^{2} y+x^{3} y+y^{2}=0$ is an algebraic solution of $F=0$. 


\section{REFERENCES}

[1] Bronstein, M., Integration of elementary functions, $J$. Symb. Comput., 9, 117-173, 1990.

[2] Bronstein, M. and Lafaille, S., Solutions of linear ordinary differential equations in terms of special functions, Proc. ISSAC2002, ACM Press, 2002.

[3] Carnicer, M.M., The Poincaré problem in the nondicritical case, Ann. of Math., 140, 289-294, 1994.

[4] Cerveau, D. and Lins Neto, A., Holomorphic foliations in $\mathbb{C} P(2)$ having an invariant algebraic curve, Ann. Inst. Fourier, 41(4), 883-903, 1991.

[5] Cormier, O., Singer, M.F., Trager, B.M. and Ulmer, F., Linear differential operators for polynomial equations, J. Symb. Comput., 34, 355-398, 2002.

[6] Cormier, O., On Liouvillian solutions of linear differential equations of order 4 and 5, Proc. ISSAC2001, 93-100, ACM Press, 2001.

[7] Corral, N. and Fernández-Sánchez, P., Isolated invariant curves of a foliation, to apper in Proc. Amer. Math. Soc..

[8] Davenport, J.H., On the integration of algebraic functions, Lecture Notes in Computer Science, 102, Springer-Verge, New York, 1981.

[9] Feng, R. and Gao, X.S., Rational general solutions of algebraic ordinary differential equations, Proc. ISSAC2004, 155-162, ACM Press, 2004.

[10] Feng, R. and Gao, X.S., A polynomial-time algorithm to compute rational solutions of first order autonomous ODEs, MM-Preprints, No.23, 54-65, December, 2004.

[11] Fulton, W., Algebraic Curves, Benjamin/Cummings Publishing Company, Inc, 1969.

[12] Hubert, E., The general solution of an ordinary differential equation, Proc. ISSAC1996, 189-195, ACM Press, 1996.

[13] Kolchin, E.R., Differential Algebra and Algebraic Groups, ACM Press, New York, 1973.

[14] Kovacic, J.J., An algorithm for solving second order linear homogeneous differential equations, J. Symb. Comput., 2(1), 3-43, 1986.

[15] Lang, S., Introduction to Algebraic and Abelian Functions, second edition, Springer-Verlag, New York, 1972.

[16] Poincaré, H., Sur l'intégration algébrique des équations différentielles du premier ordre et du premier degré, Rend. Circ. Mat. Palermo, 11, 193-239, 1897.

[17] Risch, R.H., The problem of integration in finite terms, Trans. Amer. Math. Soc., 139, 167-189, 1969.

[18] Ritt, J.F., Differential Algebra, Amer. Math. Sco. Colloquium, New York, 1950.
[19] Singer, M.F., Liouillian solutions of $n$th order homogeneous linear differential equations, Amer. J. Math., 103(4), 661-682, 1981.

[20] Singer, M.F., Liouillian first integrals of differential equations, Trans. Amer. Math. Sco., 333(2), 673-688, 1992.

[21] Singer, M.F. and Ulmer, F., Liouvillian solutions of third order linear differential equations: new bounds and necessary conditions, Proc. ISSAC1992, 57-62, ACM Press, 1992.

[22] Trager, B., Integration of Algebraic Functions, Ph.D thesis, Dpt. of EECS, Massachusetts Institute of Technology, 1984.

[23] Ulmer, F. and Calmet, J., On liouvillian solutions of homogeneous linear differential equations, Proc. ISSAC1990, 236-243, ACM Press, 1990.

[24] Van der Put, M. and Singer, M. Galois Theory of Linear Differential Equations, Springer, Berlin, 2003.

[25] von Zur Gathen, J. and Gerhard, J. (1999). Modern Computer Algebra, Cambridge University Press, Cambridge.

[26] Walker, R. J., Algebraic Curves, Princeton Unv. Press, 1950. 